\title{
Motivações na prática de exercício físico e os estágios de mudança dos estudantes no ensino superior
}

https://doi.org/10.11606/issn.1981-4690.v35i1p55-65
Fernando Marques Fernandes de Lemos* Nuno Corte-Real* Cláudia Dias* António Manuel Fonseca*
*Centro de Investig ação. Formação, Inovação e Intervenção em Desporto, Faculdade de Desporto, Universidade do Porto, Porto, Portugal.

\section{Resumo}

Este estudo procurou analisar as relações existentes entre as regulações motivacionais, os estágios de mudança e a frequência da prática de exercício dos estudantes de enfermagem, em ambos os sexos. Foi realizado um estudo transversal, descritivo e exploratório com 535 estudantes. Os resultados indicaram que: (a) 55.3\% dos estudantes não tinham uma prática de exercício de forma consistente e continuada; (b) 27.6\% mantinham um envolvimento regular nesta prática há mais de seis meses; (c) o sexo masculino, com tempo de prática superior há seis meses, apresentava valores mais elevados que o sexo feminino na sua motivação intrínseca; (d) o sexo feminino apresentava uma frequência de prática de exercício, frequente e muito frequente, menor que o sexo masculino. Os estudantes parecem relegar a prática do exercício físico para um segundo plano, tornando este assunto merecedor de uma atenção especial, inclusivamente pelas próprias instituições de ensino.

Palavras-Chave: Autodeterminação; Frequência do Exercício; Comportamentos saudáveis; Estudantes de Enfermagem.

\section{Introdução}

A importância e os benefícios da prática de exercício físico na população têm sido bem documentados, ao nível físico, psicológico e social resultando em consideráveis benefícios para a saúde física e mental, estando associada, por exemplo, a um risco mais baixo de doenças cardiovasculares, câncer, diabetes tipo 2, hipertensão arterial, obesidade, osteoporose e depressão ${ }^{1}$.

Todavia, ao contrário do que seria esperado, há evidências de que os jovens adultos entre os 18 e 25 anos, dentre eles os estudantes do ensino superior, não apresentam hábitos de uma prática esportiva regular². Por outro lado importa reconhecer que, embora não correspondam à maioria da população nesta faixa etária, os jovens adultos que frequentam o ensino superior, $\mathrm{e}$ em particular os matriculados em cursos relacionados com a área da saúde (e.g., enfermagem), poderão ser considerados, de um modo global, mais bem informados sobre os benefícios da prática de exercício físico (EF) do que aqueles que não o frequentam ${ }^{3}$.

Contudo, o estudante do ensino superior durante o seu ciclo de estudos, pode ser confrontado com o compromisso com atividades sociais e académicas, que os leva a terem uma percepçáo de falta de tempo para outras atividades, incluindo a prática de $\mathrm{EF}^{4}$. Neste caso, os comportamentos sedentários poderão ser explicados, pelo menos parcialmente, pelo fato de, no final do ensino médio, a prática de EF deixar de ser obrigatória, tornando-se uma atividade voluntária quando os estudantes dáo continuidade aos seus estudos no ensino superior, ou quando ingressam no mercado de trabalho.

Por outro lado, algumas das razóes para o baixo envolvimento dos estudantes do ensino superior no $\mathrm{EF}$, podem estar relacionados com alguns tipos de processos motivacionais extrínsecos envolvidos neste comportamento ${ }^{5}$, tendo alguns estudos demonstrado que a manutençáo do comportamento ao longo do 
tempo se relaciona de forma positiva com uma forma mais autónoma de regulaçáo motivacional, promovendo uma maior continuidade da prática de exercício físico ${ }^{6}$. Para além disso, os estudantes do ensino superior são, em primeira instância, os responsáveis pelo planejamento e execução na promoção dos seus hábitos de prática de EF. Contudo os estudantes do ensino superior não parecem estar preparados de forma eficaz para a transição para uma atividade física que, como referimos anteriormente, deixa de ser obrigatória e passa a ser voluntária ${ }^{7}$.

De fato, estes dados sobre uma baixa participação no EF também são corroborados por estudos desenvolvidos entre estudantes do ensino superior na Alemanha ${ }^{8}$ e no Brasil $^{9}$ nos quais foram identificados elevados níveis de sedentarismo em estudantes de enfermagem.

Com efeito, na literatura proliferam os modelos teóricos utilizados para explicar os comportamentos de saúde (no qual se inclui o exercício físico), entre os mais populares destacam-se o modelo transteórico (Transtheoretical Model of Change, $\left[\mathrm{TTM}^{10}\right]$ ) e a teoria da autodeterminação (Self Determination Theory, $\left.\left[\mathrm{SDT}^{11}\right]\right)$.

A conceitualização do TTM é fundamentada na premissa de que a mudança comportamental ocorre ao longo de um processo, no qual as pessoas dispóem de seis níveis de prontidão para a mudança. Estes seis níveis estão sequenciados e ordenados de maneira específica e são denominados estágios ou fases de mudança: pré-contemplação (o indivíduo não pretende mudar os seus hábitos nos próximos seis meses); contemplação (o indivíduo pretende mudar os seus hábitos nos próximos seis meses); preparação (o indivíduo pretende mudar os seus hábitos nos próximos 30 dias); ação (o indivíduo realiza de maneira deliberada um comportamento e mantém-no entre um e seis meses); manutenção (o comportamento permanece por um período superior a seis meses); e finalização (o indivíduo tem moldado o seu comportamento há pelo menos cinco anos sem recaídas, sendo o objetivo final para quem realiza mudanças em alguns tipos de comportamentos, como os hábitos tabágicos, ou consumo de álcool e outras drogas).

Já a conceitualização da SDT adota uma abordagem multidimensional do "porquê" algumas pessoas se envolverem em comportamentos de saúde positivos e adaptativos e outras não o fazerem, examinando o grau de motivaçáo (i.e., autônoma ou controlada) que uma pessoa possui relativamente a um comportamento particular.

O foco central da teoria da autodeterminação tem sido conceitualizar a motivação humana ao longo de um continuum, identificando várias formas ou estilos de regulaçáo comportamental, que variam em graus da autodeterminação ${ }^{12}$. O continuum de autodeterminação apresenta entâo regulaçóes, que váo desde as mais controladas às mais autônomas, distribuídas ao longo de seis estilos de regulação: amotivação (caracterizada pela ausência de intenção e de comportamento próativo); regulaçấo externa (a pessoa é influenciada por fatores externos, executando a ação para receber recompensas ou evitar puniçóes); regulação introjetada (os comportamentos são influenciados por pressóes internas, como a culpa, a ansiedade ou a busca de reconhecimento social); regulaçáo identificada (ocorre em situaçôes de reconhecimento e valorização, onde o indivíduo considera a atividade importante e aprecia os resultados e benefícios subjacentes ao comportamento que ela lhe pode proporcionar); regulação integrada (o comportamento torna-se totalmente integrado, incluindo náo somente a identificação com a importância do comportamento, mas também a integração dessas identificaçóes com a sua maneira de pensar); motivação intrínseca (a atividade é vista como um fim em si mesma e é praticada por conta do prazer, da satisfaçáo e do divertimento que ela proporciona). De fato ambos os modelos teóricos têm sido usados de maneira proeminente para explicar o comportamento da prática de exercício físico ${ }^{13}$.

Portanto, no que se refere ao EF, por um lado, o TTM pressupóe uma perspectiva mais "quantitativa" sobre a motivação, sustentando que os indivíduos que estão em estágios de ação e manutenção são mais motivados do que aqueles que se encontram em précontemplação e contemplação; por outro lado, a SDT dá destaque à "qualidade" desta mesma motivação, sugerindo que o envolvimento na prática de EF pode ser regulado por razóes mais ou menos autônomas ou controladas; neste caso é frequente que a participação na prática de EF seja mantida ao longo do tempo somente quando as regulaçóes autônomas são evidenciadas.

Importa ainda salientar que a aplicação destes modelos teóricos em Portugal, na área da saúde, tem conduzido à realização de importantes estudos longitudinais e experimentais que comprovam a utilidade destes referenciais teóricos para o delineamento de intervençôes práticas de prevenção e promoção da saúde ${ }^{14-15}$.

Considerando as investigaçóes que procuram 
conhecer e compreender a realidade que está inerente aos hábitos de práticas de esportes e de exercício físico em estudantes do ensino superior, o presente estudo pretendeu analisar as relaçóes existentes

\section{Método}

Este estudo caracteriza-se por ter um delineamento do tipo transversal, descritivo e exploratório. Para ser incluído no estudo o participante deveria atender aos seguintes critérios: idade igual ou superior a 18 anos e estar devidamente matriculado no curso de enfermagem. Foram excluídos os indivíduos que apresentassem restriçóes médicas para a prática de exercícios físicos regulares. Participaram neste estudo 535 estudantes de ambos os sexos. No ano letivo em apreciação (2015), estiveram matriculados, nos diversos anos curriculares 1196 estudantes, 1012 (84.6\%) do sexo feminino 184 (15.4\%) do sexo masculino. A amostra correspondeu a 45\% do universo estudado, todos os estudantes frequentavam a Escola Superior de Enfermagem do Porto, Portugal.

\section{Instrumentos de Medida}

Stage of Exercise Behaviour Change Questionnaire. Para avaliar o posicionamento do estudante em função do estágio de mudança, foi utilizado o Stage of Exercise Behaviour Change Questionnaire, a versão traduzida e adaptada para a realidade portuguesa ${ }^{16}$ do Stage of Exercise Behaviour Change Questionnaire ${ }^{17}$.

Este instrumento refere-se as medidas psicológicas que foram desenvolvidas para o exercício físico, trata-se do questionário adaptado e derivado do comumente chamado estágios de mudança (i.e., TTM) e é constituído por uma questáo inicial ("Faz algum tipo de exercício físico com regularidade de no mínimo três vezes por semana entre 20 e 60 minutos?"), relativamente à qual os respondentes, devem selecionar, entre cinco afirmaçôes disponíveis, a que entendem mais se aproximar da sua situação atual: (a) "Não faço exercício físico com regularidade nem pretendo começar nos próximos 6 meses." (pré contemplação); (b) "Não faço exercício físico com regularidade mas pretendo começar nos próximos 6 meses." (contemplação); (c) "Não faço exercício físico com regularidade mas pretendo começar nos próximos 30 dias." (preparação); (d) "Faço exercício físico com regularidade há menos de 6 meses." entre as regulaçóes motivacionais, os estágios de mudança e a frequência da prática de exercício físico dos estudantes do curso de enfermagem, em ambos os sexos.

(ação); e (e) "Faço exercício físico com regularidade há mais de 6 meses" (manutenção).

Behavioral Regulation in Exercise Questionnaire - 2 - BREQ2. Para avaliar o comportamento no exercício foi utilizado o Behavioral Regulation in Exercise Questionnaire - 2-BREQ2, a versão traduzida e adaptada para a realidade portuguesa ${ }^{18}$ do Behavioral Regulation in Exercise Questionnaire - 2-BREQ2 ${ }^{19}$.

Este instrumento é constituído por 19 afirmaçóes sobre as várias formas de regulação comportamental associadas ao exercício, relativamente a cada uma das quais os respondentes devem indicar em que medida a consideram como importante para a sua decisáo de praticar, ou nâo, exercício, através de uma escala do tipo Likert de 5 pontos $(0$ = "Não é verdade para mim" a 4 = "Muitas vezes é verdade para mim"). As referidas 19 afirmações deste instrumento distribuem-se por cinco fatores: (i) amotivação (e.g., "Acho que o exercício é uma perda de tempo."); (ii) regulação externa (e.g., "Faço exercícios porque outras pessoas dizem que devo fazer."); (iii) regulação introjetada (e.g., "Sinto-me culpado/a quando não faço exercícios."); (iv) regulação identificada (e.g., "Dou valor aos benefícios/vantagens dos exercícios."); e (v) motivação intrínseca (e.g., "Gosto das minhas sessões de exercícios.").

Adicionalmente, os estudantes responderam a um conjunto de questóes sobre variáveis sociodemográficas (e.g., idade, sexo). $\mathrm{Na}$ avaliação da frequência da prática de $\mathrm{EF}$, os estudantes responderam à seguinte questão: "Numa semana normal, com que frequência pratica algum tipo de exercício fisico?', podendo selecionar uma de nove afirmaçóes disponíveis: (a) nenhuma vez por semana; (b) menos de uma vez por semana; (c) uma vez por semana; (d) duas vezes por semana; (e) três vezes por semana; (f) quatro vezes por semana; (g) cinco vezes por semana; (h) seis vezes por semana; e (i) todos os dias.

\section{Procedimentos}

O projeto de pesquisa foi aprovado pelo Comitê de Ética em Pesquisa em Seres Humanos da Faculdade de Desporto da Universidade do Porto (CEFADE 21/2016). 
Para a coleta dos dados os estudantes preencheram um questionário presencialmente, e em horário regular das aulas. Adicionalmente, os estudantes preencheram um formulário onde declararam aceitar a participação voluntária no respectivo estudo, sendo também informados que o anonimato estaria assegurado.

\section{Análise dos dados}

As respostas da frequência da prática de $\mathrm{EF}$ foram agrupadas em quatro categorias: (i) inexistente (nenhuma vez por semana); (ii) pouco frequente (menos de uma vez por semana até duas vezes por semana); (iii) frequente (três vezes por semana), e, (iv) muito frequente (mais de três vezes por semana), esta escolha fundamenta-se nos critérios da frequência da prática de EF mínima necessária, segundo a posição do Colégio Americano de Medicina Esportiva ${ }^{20}$.

Adicionalmente, como um número limitado de estudantes estava no estágio de pré-contemplação, os seus dados foram combinados com os dos estudantes do estágio de contemplação para formar um único

\section{Resultados}

Participaram neste estudo 535 estudantes de ambos os sexos, 459 (85.8\%) eram do sexo feminino e $76(14.2 \%)$ do sexo masculino, com idades compreendidas entre os 18 e os 32 anos e média de idades de $19.75(\mathrm{DP} \pm 1.95)$ anos.

\section{Frequência de prática de exercício físico}

Quanto à frequência da prática, 27.6\% dos participantes deste estudo foram categorizados com uma prática de EF inexistente e 45.2\% com uma prática de EF pouco frequente; apenas $27.2 \%$ tinham uma frequência de prática de EF de no mínimo três vezes por semana.

A análise da frequência do EF em função do sexo não revelou a existência de diferenças estatisticamente significativa entre as variáveis $\left(\chi 2_{[3,535]}=9.71, p \geq .05\right)$. Além disso, uma análise mais pormenorizada dos dados revelou que todas as categorias de frequência de EF se apresentavam muito similares em ambos os sexos, apesar do sexo masculino, de maneira residual, apresentar valores porcentuais superiores nas categorias estágio denominado "pré-preparação". Para além disso, a combinação de participantes nestes estágios de mudança iniciais é uma abordagem que já foi utilizada no passado ${ }^{21}$.

O tratamento estatístico ${ }^{22}$ incluiu análises descritivas de frequência e porcentagem nas variáveis sexo, estágios de mudança e frequência da prática de EF. Para identificar possíveis diferenças entre os sexos na frequência de EF nos estágios de mudança foi utilizado o teste do qui-quadrado $(\chi 2)$, recorrendo-se à análise dos resíduos ajustados estandardizados que, em módulo, eram superiores a 1.96 para $p<.05$, indicando assim as células que se afastavam da hipótese nula de independência. Para além disso, foi utilizada a ANOVA fixando-se o nível de significância em $p<$ .05, bem como o teste post hoc de Scheffé, no sentido de examinar as possíveis diferenças existentes nas diferentes regulaçóes motivacionais entre os estágios de mudança e entre a frequência da prática de EF dos estudantes, em ambos os sexos. Por fim, para examinarmos as correlaçóes entre as categorias da frequência de EF e os estágios de mudança do exercício recorremos a análise de correlações de Spearman.

frequente e muito frequente de EF. (TABELA 1).

\section{Estágios de Mudança}

No que concerne aos estágios de mudança, nomeadamente aos hábitos da prática de EF, importa ressaltar que $55.3 \%$ dos estudantes não tinham uma prática de EF de forma consistente e continuada, estando inseridos nas fases iniciais dos estágios de mudança (i.e., pré-preparação e preparação). Destes, $27.3 \%$ não manifestavam qualquer intenção de se envolver na prática de EF pelo menos nos três meses seguintes; em contraponto, $27.6 \%$ da amostra global dos estudantes tinham uma prática de EF continuada durante mais de seis meses (fase de manutenção). Os resultados revelaram ainda uma associação estatisticamente significativa entre os estágios de mudança e o sexo $\left(\chi 2_{[3,535]}=13.56, p \leq .005\right)$. O estudo através dos resíduos ajustados estandardizados revelou que havia menos estudantes do sexo masculino no estágio de ação e mais no estágio de manutenção do que os esperados. 
TABELA 1 - Caracterização dos estudantes em função da frequência de EF em ambos os sexos.

\begin{tabular}{lccc}
\hline Frequência de EF & $\begin{array}{c}q(\mathbf{n}=\mathbf{4 5 9}) \\
\mathbf{\%}\end{array}$ & $\begin{array}{c}\hat{(\mathbf{n}=76)} \\
\mathbf{\%}\end{array}$ & $\begin{array}{c}\text { Total }(\mathbf{N}=\mathbf{5 3 5}) \\
\mathbf{\%}\end{array}$ \\
\hline $\begin{array}{l}\text { Inexistente } \\
\text { Pouco Frequente }\end{array}$ & 26.4 & 28.9 & 27.6 \\
Frequente & 48.1 & 42.2 & 45.2 \\
Muito Frequente & 17.2 & 19.7 & 18.4 \\
& 8.3 & & 8.8 \\
\%Total & $100 \%$ & $100 \%$ & $100 \%$ \\
\hline
\end{tabular}

Já nos estudantes do sexo feminino acontecia o inverso: havia um maior número de estudantes no estágio de ação e um menor número no estágio da manutenção do que as esperadas. Os resultados indicaram ainda que os estudantes do sexo feminino se mostravam mais disponíveis no que se refere a tomarem medidas orientadas para se envolverem na prática de EF de maneira imediata (fase de preparação); por outro lado $35.5 \%$ dos estudantes do sexo masculino mantinham um comportamento estável e regular de prática desportiva há mais de seis meses (TABELA 2).

TABELA 2 - Caracterização dos estudantes em função dos estágios de mudança e respectivos resíduos ajustados, em ambos os sexos.

\begin{tabular}{|c|c|c|c|}
\hline \multirow{2}{*}{ Estágios de mudança } & $q(\mathrm{n}=459)$ & $\partial(n=76)$ & Total $(\mathrm{N}=535)$ \\
\hline & \% (Res. Ajust.) & \% (Res. Ajust.) & $\%$ \\
\hline Pré-preparação & 25.6 & 29.0 & 27.3 \\
\hline Preparação & 30.9 & 25.0 & 28.0 \\
\hline Ação & $23.7(2.6)$ & $10.5(-2.6)$ & 17.1 \\
\hline Manutenção & $19.8(-3.1)$ & $35.5(3.1)$ & 27.6 \\
\hline$\%$ Total & $100 \%$ & $100 \%$ & $100 \%$ \\
\hline
\end{tabular}

\section{Regulações motivacionais e estágios de mudança}

No que se refere à relação entre os estágios de mudança e as regulaçóes motivacionais, a análise do resultado do sexo masculino revelou a existência de diferenças estatisticamente significativas na regulação identificada e na motivação intrínseca nos diferentes estágios de mudança. Já no sexo feminino as diferenças estatisticamente significativas encontravam-se na regulação introjetada, na regulação identificada e na motivação intrínseca nos diferentes estágios de mudança. Os testes post hoc de Scheffé indicaram que, no sexo masculino, as diferenças significativas na regulação identificada e na motivação intrínseca, eram entre os estágios de mudança da preparação e manutenção $(p \leq .001)$ e entre os estágios de preparação e manutenção ( $p$ $\leq .001)$ respectivamente. Já no sexo feminino, as diferenças significativas na regulação introjetada e regulação identificada eram entre os estágios de ação e manutenção $(p \leq .05)$ e entre os estágios de pré preparação e manutenção $(p \leq .001)$ respectivamente (TABELA 3). 
TABELA 3 - Diferenças entre o grupo dos estágios de mudança nas diferentes regulações motivacionais dos estudantes, em ambos os sexos.

Nota:

$\mathrm{M}=$ média;

$\mathrm{DP}=$ desvio padrão;

$\mathrm{F}=$ Anova;

$p=$ valor de prova;

${ }^{* * *} p \leq .001$;

${ }^{* *} p \leq .005$.
Nota:

$M=$ média;

$\mathrm{DP}=$ desvio padrão;

$\mathrm{F}=$ Anova;

$\mathrm{p}=$ valor de prova

${ }^{* * *} p \leq .001$;

${ }^{* *} p \leq .005$.

\begin{tabular}{|c|c|c|c|c|c|c|c|}
\hline \multirow{4}{*}{ Masculino } & Amotivação & $0.26 \pm 0.43$ & $0.26 \pm 0.58$ & $0.28 \pm 0.69$ & $0.04 \pm 0.17$ & 1.37 & .242 \\
\hline & Externa & $0.43 \pm 0.54$ & $0.39 \pm 0.52$ & $0.56 \pm 0.67$ & $0.25 \pm 0.49$ & 1.18 & .322 \\
\hline & Introjetada & $1.02 \pm 0.53$ & $1.07 \pm 0.78$ & $1.25 \pm 0.74$ & $1.35 \pm 0.75$ & 1.33 & .225 \\
\hline & Intrínseca & $2.76 \pm 0.85$ & $2.67 \pm 1.11$ & $3.50 \pm 0.74$ & $3.52 \pm 0.45$ & 2.25 & $.019^{* * *}$ \\
\hline \multirow[b]{3}{*}{ Feminino } & Amotivação & $0.19 \pm 0.44$ & $0.17 \pm 0.45$ & $0.04 \pm 0.14$ & $0.01 \pm 0.10$ & 3.31 & .051 \\
\hline & Externa & $0.42 \pm 0.52$ & $0.44 \pm 0.56$ & $0.41 \pm 0.53$ & $0.35 \pm 0.57$ & 1.31 & .212 \\
\hline & Introjetada & $1.08 \pm 0.85$ & $1.32 \pm 0.85$ & $1.58 \pm 0.99$ & $1.17 \pm 0.92$ & 2.83 & $.000^{* *}$ \\
\hline
\end{tabular}

Regulações motivacionais e a frequência de exercício físico

No que concerne à relação entre as categorias da prática de EF e as regulaçôes motivacionais, a análise dos resultados do sexo masculino revelou a existência de diferenças estatisticamente significativas na regulação identificada e na motivação intrínseca, nos diferentes níveis de prática de EF. De igual modo, no sexo feminino havia diferenças nas regulaçóes introjetada, identificada e intrínseca em função da frequência de prática das estudantes (TABELA 4).

Os testes post hoc de Scheffé indicaram que, no sexo masculino, na regulação identificada e na motivação intrínseca, as diferenças significativas eram entre a prática de EF inexistente e frequente $(p \leq .05)$ e muito frequente $(p \leq .001)$. Já no sexo feminino, na regulação identificada, na regulação introjetada e na motivação intrínseca as diferenças estatisticamente significativas eram entre a prática de EF inexistente e frequente $(p \leq .001)$ e muito frequente $(p \leq .001)$.

TABELA 4 - Diferenças entre os grupos da frequência da prática de exercício físico nas diferentes regulações motivacionais dos estudantes, em ambos os sexos.

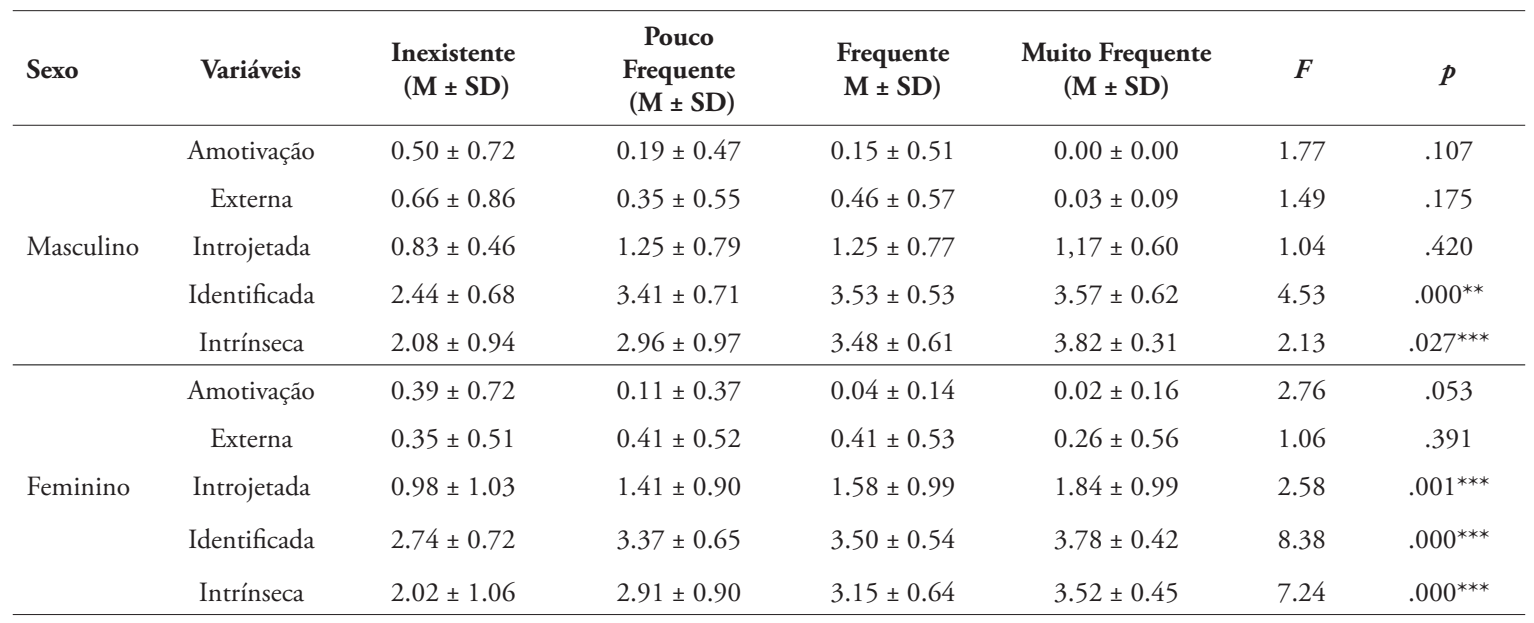


Correlações entre a frequência de exercício físico e os estágios de mudança

A análise da correlação entre as categorias da frequência de EF e os estágios de mudança do exercício revelou uma correlação positiva e significativa $(\mathrm{r}=.83 ; p \leq .001)$. Ou seja, o EF frequente e muito frequente correlacionava-se positivamente com os estágios de mudança de ação e manutenção (FIGURA 1).

FIGURA 1 - Correlação entre o grupo dos estágios de mudança e o grupo da frequência de prática de EF.

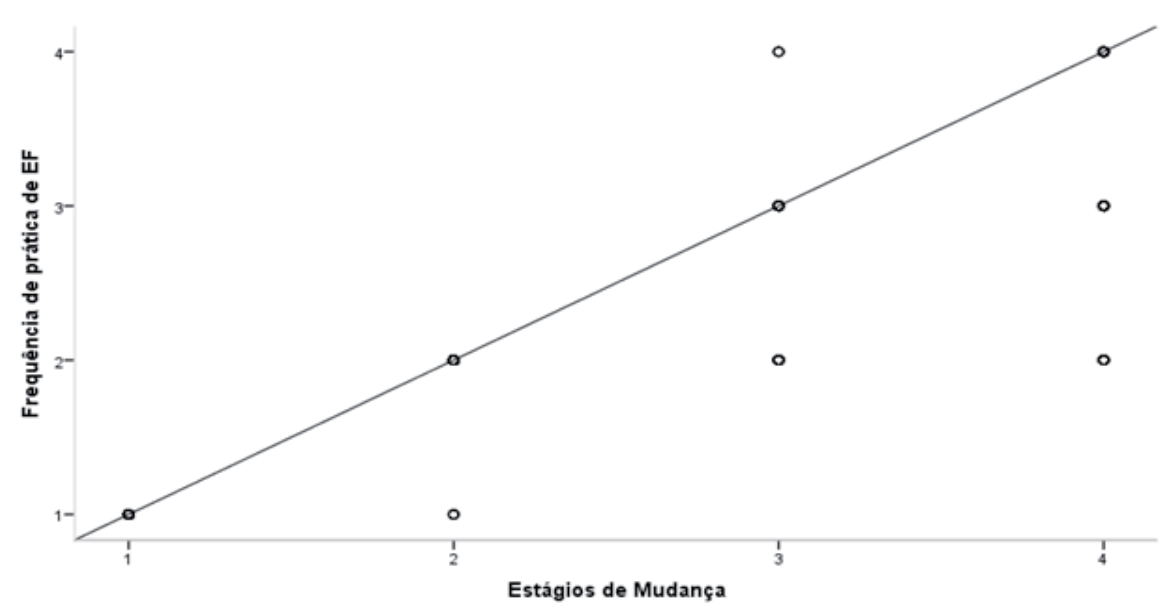

Nota:

Frequência de EF: $1=$ Inexistente, $2=$ Pouco Frequente, 3= Frequente, 4= Muito Frequente.

Estágios de mudança: 1= Pré-preparação; 2= Preparação, 3= Ação, 4= Manutenção

\section{Discussão}

O objetivo deste estudo foi analisar as relaçóes existentes entre as regulaçóes motivacionais, os estágios de mudança e a frequência da prática de exercício físico, em estudantes de enfermagem de ambos os sexos.

Relativamente aos estágios de mudança, mais de metade dos participantes reportaram não estar envolvidos numa prática consistente e continuada de EF. Os dados relativos à frequência de prática de EF confirmaram esta informação, mostrando resultados nada animadores: mais de um quarto dos estudantes não praticava EF e quase metade apresentava uma frequência de prática de EF aquém do desejável. Para além disso, pouco mais de um quarto dos estudantes relataram uma prática de EF de modo frequente e/ou muito frequente, revelando assim sua pouca expressão junto dos estudantes de enfermagem. Este panorama não é nesta população, já que os nossos resultados também são similares aos obtidos num estudo anterior alargado com universitários de 23 países $^{23}$, onde foi identificada em uma proporção substancial de estudantes uma participação no EF abaixo daqueles níveis recomendados.

Por outro lado, a análise da frequência da prática de EF em função do sexo revelou resultados muito semelhantes, em ambos os sexos, tanto nos estudantes que relataram praticar EF de maneira frequente e muito frequente. Contudo, ressalte-se que apesar de não existirem diferenças significativas o sexo masculino apresentava, de maneira geral, porcentagens de participação superiores no EF frequente e muito frequente.

Relativamente à relação entre as regulações motivacionais e a prática de EF, era esperado que os estudantes mais autodeterminados apresentassem uma prática de EF frequente e regular. Em consonância com essa expectativa, os resultados do presente estudo revelaram que estudantes com valores mais elevados de regulaçôes autónomas apresentavam, de uma forma geral, frequência mais elevada de prática de EF e um envolvimento mais consistente e continuado nessa prática. Os nossos resultados são congruentes 
com os modelos teóricos da SDT, do TTM e com outros estudos ${ }^{24-25}$, onde a participação dos indivíduos numa prática de EF e a permanência nesta prática, de maneira regular e estável no tempo, estava relacionada diretamente com a sua motivação.

Com efeito, no seu conjunto, as regulaçôes mais autodeterminadas eram significativamente superiores nos estudantes que estavam em fases mais avançadas dos estágios de mudança (ação e manutenção), os quais assumiam uma prática de $\mathrm{EF}$ frequente ou muito frequente, comparativamente àqueles que estavam nos estágios iniciais (pré-preparação e preparação). Além disso, as regulaçóes motivacionais relativas à motivação intrínseca (a atividade é vista como um fim em si mesma), regulação identificada (o indivíduo considera a atividade importante e aprecia os resultados e benefícios), e, em menor medida, à regulação introjetada (os comportamentos são influenciados por pressões internas), estavam correlacionadas significativamente com os estudantes que reportaram uma maior frequência da prática de EF.

De fato, nossos resultados corroboram outros estudos que, ao analisarem as motivaçóes da prática de EF a partir da SDT e do TTM, verificaram que os participantes com um perfil mais autodeterminado encontravam-se nos estágios de mudança da ação e manutenção ${ }^{26,27}$.

Ainda relativamente à amostra global dos estudantes, a permanência na prática de EF é um comportamento que tende a ser mantido com um interesse maioritariamente autodeterminado (i.e., pela regulação identificada e motivação intrínseca). Para além da motivação intrínseca para a participação no EF, o comportamento do estudante de enfermagem também tem apoio na proposição da $\mathrm{SDT}^{28}$, realçando a importante contribuiçấo de outros tipos de regulaçóes autodeterminadas (i.e., regulaçáo identificada) e, em menor medida, da regulação introjetada, regulaçóes com potencial para favorecer comportamentos positivos de promoção da saúde, como também um maior envolvimento e consequente adesão aos mesmos.

No entanto, em decorrência destas análises também é plausível, em nossa opinião, que a regulação identificada (regulação que valoriza os benefícios do $\mathrm{EF})$, tenha influência nas respostas dos estudantes de enfermagem, os quais, pertencendo a um curso da área da saúde, estariam mais sensibilizados para os benefícios relacionados com a prática de EF (independente de praticarem ou não EF).

Podemos especular que, em nosso estudo, os resultados das respostas poderiam estar, em certa medida, enviesados visto os estudantes pertencerem a um curso da área da saúde. De fato, os estudantes poderiam não ter nenhum envolvimento ou frequência na prática de $\mathrm{EF}$, mas considerarem e percepcionarem esta prática de igual forma importante, valorizando em suas respostas as afirmaçóes que estariam associadas à uma regulação identificada (e.g., "Penso que é importante fazer um esforço por fazer exercício regularmente."). Ou seja, a prática de EF poderia ser percepcionada pelo estudante, em seu quotidiano, como uma prática desejável e saudável, ainda que não fosse realizada regularmente pelo mesmo. Nesse contexto, teríamos então uma fonte de motivação autodeterminada, baseada em medidas de autorrelato, mas que estariam relacionadas apenas com a percepção do estudante sobre a prática de $\mathrm{EF}$ e não com o seu comportamento (i.e., sua frequência e participação no $\mathrm{EF}$ ).

Por outro lado, a análise dos estágios de mudança reportados pelos estudantes, em ambos os sexos, revelaram que as regulaçôes motivacionais estavam em consonância com os estágios de mudança. Para além disso, no sexo masculino as regulaçóes motivacionais que apresentaram ser significativas nos estágios de mudança e na frequência do EF foram a regulação identificada e a motivação intrínseca; já no sexo feminino, tanto nos estágios de mudança como na frequência do $\mathrm{EF}$, as regulaçóes motivacionais significativas foram a regulação introjetada, a regulação identificada e a motivação intrínseca. Tendo em consideração esta análise, a motivação intrínseca conjuntamente com a regulação identificada e, em menor medida, a regulação introjetada, constituíram-se como as variáveis que mais se relacionam com os estágios da ação e manutenção e a prática de $\mathrm{EF}$ de um modo frequente e muito frequente nos estudantes de enfermagem.

A motivação mais autodeterminada, porque apresenta uma maior correlação com os estágios de mudança da manutenção e ação e com uma maior frequência da prática de EF, parece ser, de fato, um indicador da frequência regular da prática de $\mathrm{EF}$ nos estudantes de enfermagem. Uma maior adesão à prática de $\mathrm{EF}$ é o resultado de um comportamento mais autônomo e portanto mais autodeterminado, revelado aqui como importante a uma prática de EF continuada em períodos superiores a seis meses.

Nossos resultados, apoiados nos estudos já descritos, apontam para que o próximo passo seja examinar as interdependências entre as regulaçóes motivacionais e frequência da prática de EF ao longo do tempo. Para além disso, um estudo de design longitudinal proporcionaria uma leitura dos resultados que 
permitisse delinear estratégias mais apropriadas para uma intervenção futura na integração da mudança de comportamentos prevista já teoricamente ${ }^{29}$. Adicionalmente consideramos constituir-se como importante, em futuros estudos, que se opte também por investigar mais profundamente a magnitude e o impacto das regulaçóes motivacionais na adesão ao EF, utilizando outras variáveis (e.g., intensidade e duração do exercício físico).

$\mathrm{O}$ nosso estudo aponta que os estudantes de enfermagem, parecem relegar a prática do EF para um segundo plano, apesar de parecer que estão bem informados quando aos benefícios da sua prática. Este comportamento alerta-nos para que deveriam ser repensadas alternativas, impedindo que a possível gravidade resultante deste panorama possa potenciar futuramente um impacto negativo na saúde desta população, o que por si só torna este assunto merecedor de uma atenção especial, inclusivamente pelas próprias instituiçóes de ensino. Assim, parecenos urgente pensar-se em projetos de intervenção que possibilitem a estes jovens transformar os seus conhecimentos em comportamentos saudáveis, como é o caso do exercício físico.

\title{
Agradecimentos
}

Agradecemos a Fundação Para Ciência e a Tecnologia (FCT) pelo financiamento concedido para o pesquisador Fernando Marques Fernandes de Lemos.

\begin{abstract}
Motivations in the practice of physical exercise and the stages of student change in higher education

This study sought to analyze the relationships between motivational regulations, the stages of change and the frequency of nursing students' exercise practice in both sexes. A cross-sectional, descriptive and exploratory study was carried with 535 students. The results indicated that: (a) 55.3\% of students did not practice consistently and continuously; (b) 27.6\% had a regular involvement in this practice for more than six months; (c) males, who had been in practice for more than six months, had higher values than the female gender in the intrinsic motivation; (d) females had a frequency of frequent and very frequent exercise, which was lower than that of males. Students seem to relegate the practice of physical exercise to a secondary level, making this subject worthy of special attention, even by the educational institutions themselves.
\end{abstract}

KeYWORDS: Self-determination; Frequency of Exercise; Healthy behaviors; Nursing students.

\section{Referências}

1. Ekelund U, Ward HA, Norat T, et al. Physical activity and all-cause mortality across levels of overall and abdominal adiposity in European men and women: the European Prospective Investigation into Cancer and Nutrition Study (EPIC). Am J Clin Nutr. 2015;101:613-621.

2. Craig L, Lambert E. The pandemic of physical inactivity: global action for public health. Lancet. 2012;380:294-305.

3. Ann-Stark M, Manning-Walsh J, Vliem S. Caring for self while learning to care for others: a challenge for nursing students. J Nurs Educ. 2005;44:266-280.

4. Díez S, Pérez-Fortis A. Socio-demographic predictors of health behaviors in Mexican college students. Health Promot Inter. 2010;25:85-93.

5. Ryan R, Deci E. On happiness and human potentials: A review of research on hedonic and eudaimonic well-being. Ann Rev Psychol. 2001;52:141-166.

6. Markland D, Tobin V. Need support and behavioural regulation for exercise among exercise referral scheme clients. The mediating 
role of psychological need satisfaction. Psychol Sport Exerc. 2010;11:91-99.

7. Corte-Real N, Dias C, Corredeira R, Barreiros A, Bastos T, Fonseca AM. Prática desportiva de estudantes universitários: o caso da Universidade do Porto. Rev Port Cien Desp. 2008;8:219-228.

8. Lehmann F, von Lindeman K, Klewer J, Kugler J. BMI, physical inactivity, cigarette and alcohol consumption in female nursing students: a 5-year comparison. BMC Med Educ. 2014;14:1-6

9. Pires CG, Mussi FC, Cerqueira BB, Pitanga FJ, Silva DO. Physical activity practice among undergraduate students in nursing. Acta Paul Enferm. 2013;26:436-443.

10. Prochaska J, Norcross J, Diclemente C. Applying the stages change. In: Gerald P, Norcross J, Greene B, editores. Psychologists Desk Reference. Oxford: Oxford University Press; 2013. p. 310-315.

11. Deci E, Richard R. Intrinsic motivation and self-determination in human behavior. New York: Springer Science and Business Media; 1985.

12. Fernandez H, Vasconcelos-Raposo J. Validación e aplicación de modelos teóricos motivacionales en el contexto de la educación física. Cuad Psicol Dep, 2004;4:67-89.

13. Plotnikoff RC, Costigan SA, Karunamuni N, Lubans DR. Social cognitive theories used to explain physical activity behavior in adolescents: a systematic review and meta-analysis. Prev Med. 2013;56:245-253.

14. Silva M, Barata T, Teixeira P. Exercício físico na diabetes: missão impossível ou uma questão de motivação? Rev Port Card. 2013;32:35-43.

15. Teixeira P, Silva M, Matta J, Palmeira A, Markland D. Motivation, self-determination and long-term weight control. Int J Behav Nutr Phy. 2012;9:1-13.

16. Mendes MJ, Corte-Real N, Dias C, Fonseca AM. Excesso de peso e obesidade na escola: conhecer para intervir. Rev Port Cien Desp. 2014;14:77-92.

17. Marcus BH, Selby VC, Niaura RS, Rossi JS. Self-efficacy and the stages of exercise behavior change. Res Q Exerc Sport. 1992;63:60-66.

18. Palmeira A, Teixeira P, Silva M, Markland D. Confirmatory factor analysis of the behavioural regulation in Exercise Questionnaire - Portuguese Version. European Congress of Sport Psychology; 2007 Sep 4-9; Halkidiki, Greece. Halkidiki: FESPAC: 2007.p.87. Available from: https://www.researchgate.net/publication/256090468_Confirmatory_ Factor_Analysis_of_the_Behavioural_Regulation_in_Exercise_Questionnaire_-_Portuguese_Version

19. Markland D, Tobin V. A modification to the behavioural regulation in exercise questionnaire to include an assessment of amotivation. J Sport Exerc Psy. 2004;26:191-196.

20. Riebe D, Franklin BA, Thompson PD, et al. Updating ACSM Recommendations for exercise preparticipation health screening. Med Sci Sports Exerc. 2015;47:2473-2479.

21. Daley D, Duda J. Self-determination, stage of readiness to change for exercise, and frequency of physical activity in young people. Eur J Sport Sci. 2006;6:231-243.

22. Field, A. Discovering statistics using IBM SPSS statistics. London: Sage Publications Ltd; 2013.

23. Haase A, Steptoe A, Sallis JF, Wardle J. Leisure-time physical activity in university students from 23 countries: associations with health beliefs, risk awareness, and national economic development. Prev Med. 2004;39:182-190.

24. Rouse P, Ntoumanis N, Duda J. Effects of motivation and depletion the ability to resist the temptation to avoid physical activity. Int J Sport Exerc Psychol. 2013;11:39-56.

25. Standage M, Gillison F, Ntoumanis N, Treasure D. Predicting students' physical activity and health-related well-being: A prospective cross-domain investigation of motivation across school physical education and exercise settings. J Sport Exerc Psychol. 2012;34:37-60.

26. Matsumoto H, Takenaka K. Motivational profiles and stages of exercise behavior change. Int J Sport Health Sci. 2004;2:89-96.

27. Buckwort J, Lee R, Regan G, Schneider L, Diclemente C. Decomposing intrinsic and extrinsic motivation for exercise: Application to stages of motivational readiness. Psychol Sport Exerc. 2007;8:441-461.

28. Deci E, Ryan R. The" what" and" why" of goal pursuits: Human needs and the self-determination of behavior. Psychol Inq. 2001;11:227-268.

29. Deci E, Ryan R. Self-determination theory: A macrotheory of human motivation, development, and health. Can Psychol. 2008;49:182-184. 
Motivações para o exercício físico no ensino superior

ENDEREÇO

Fernando Marques Fernandes de Lemos Centro de Investigação, Formação, Inovação e Intervenção em Desporto

Faculdade de Desporto

Universidade do Porto

Rua Dr. Plácido Costa, 91

4200-450 - Porto - Portugal

Submetido: 01/ 12/2018

Revisado: 09/08/2018

E-mails: fernandolemos@portugalmail.com Aceito: 26/09/2018 